\title{
MODELING AND EXPERIMENTAL MEASUREMENT WITH SYNCHROTRON RADIATION OF RESIDUAL STRESSES IN LASER METAL DEPOSITED TI-6AL-4V
}

\author{
Andreas Lundbäck ${ }^{1}$, Robert Pederson ${ }^{1,2,6}$, Magnus Hörnqvist Colliander ${ }^{2,6}$, Craig Brice ${ }^{3}$, Axel Steuwer ${ }^{4}$, Almir Heralic ${ }^{2}$, Thomas Buslaps ${ }^{5}$ \\ and Lars-Erik Lindgren ${ }^{1}$ \\ ${ }^{1}$ Luleå University of Technology, 97187 Luleå, Sweden, andreas.lundback@1tu.se \\ ${ }^{2}$ GKN Aerospace Engine Systems, 46181 Trollhättan, Sweden \\ ${ }^{3}$ NASA Langley Research Center, Hampton, Virginia 23681 USA \\ ${ }^{4}$ MAX IV Laboratory, 22100 Lund, Sweden \\ ${ }^{5}$ ID15A, European Synchrotron Radiation Facility ESRF, 38042 Grenoble, France \\ ${ }^{6}$ Chalmers University of Technology, 41296 Göteborg, Sweden
}

Keywords: Additive manufacturing, Residual stress, Synchrotron X-ray diffraction, Finite element simulation

\begin{abstract}
There are many challenges in producing aerospace components by additive manufacturing (AM). One of them is to keep the residual stresses and deformations to a minimum. Another one is to achieve the desired material properties in the final component. A computer model can be of great assistance when trying to reduce the negative effects of the manufacturing process. In this work a finite element model is used to predict the thermo-mechanical response during the AM-process. This work features a physically based plasticity model coupled with a microstructure evolution model for the titanium alloy Ti-6Al-4V. Residual stresses in AM components were measured non-destructively using high-energy synchrotron X-ray diffraction on beam line ID15A at the ESRF, Grenoble. The results are compared with FE model predictions of residual stresses. During the process, temperatures and deformations was continuously measured. The measured and computed thermal history agrees well. The result with respect to the deformations agrees well qualitatively. Meaning that the change in deformation in each sequence is well predicted but there is a systematic error that is summing so that the quantitative agreement is lost.
\end{abstract}

\section{Introduction}

Within aerospace component manufacturing, fabrication has been recognized as an efficient way to reduce the weight and lead time of complex components typically manufactured using traditional techniques, such as one-piece castings or forgings. A fabricated structure is built by joining smaller subcomponents with a possibility to combine several material forms and alloys. The weight reduction potential comes from an efficient use of different mechanical properties and achievable geometrical tolerances for castings, forgings and sheet material. An important technology in this concept is additive manufacturing (AM), which enables features such as bosses and flanges to be added on subcomponents of fabricated structures and thereby minimizes the material usage (e.g. smaller forging envelope) and shortens the lead time. The use of AM also enables changes late in the design process. It further has a great potential in the aftermarket for repair and modifications.

Additive manufacturing is a broad definition that includes several different processes and methods in which metallic material is melted in a layer-by-layer manner to form 3 dimensional geometries, either as a near net-shape part or as part of a larger structure. One important advantage with the AM method, compared to conventional manufacturing methods is the potential to significantly lower the buy-to-fly ratio, i.e. components can be manufactured with near final shape without significant machining. And because machining costs are a significant part of the total product manufacturing cost, AM therefore enables significant cost savings for both manufacturing and product pricing.

During the last few years, GKN AES in Trollhättan has been developing AM utilizing a high power laser as the heat source and metal wire as the filler material. As a result, the technology has reached a high level of maturity and is today implemented in new manufacturing. The current work focuses on exploring the Laser Metal Deposition (LMD) process of Ti-6Al-4V (Ti-64). Here a laser energy beam is used to melt the metal wire of Ti-64, layer by layer, until the appropriate geometry is built. The cooling rate of the deposited material determines the microstructure and thus also the average mechanical properties of the as built part. The substrate was instrumented with thermocouples and a LVDTgauge (Linear Variable Differential Transformer) to measure the transient temperature and deformation of the substrate during the AM-process. The measurements are thereafter used for validation of the computational model. The residual stresses are measured by means of x-ray diffraction at the ID15A beam line in the synchrotron radiation facility at ESRF in Grenoble, France. These experimentally measured residual stresses are then compared with those predicted by the simulation model.

\section{Experimental Setup}

The process that is used to add the material to the substrate is LMD-w. A part is made by melting the wire additive into beads, which can be deposited side by side and layer upon layer, se Figure 1. The beads are generated by relative motion of the deposition tool and the substrate material. At GKN Aerospace the motion of the deposition tool is generated by a 6 -axis industrial robot arm. The substrate is placed either on a static podium or on a 2-axis manipulator. Instead of a robot arm, a gantry system can also be used. The heat source is a high power (several kW range) solid state laser generating a few millimeters wide beam. 


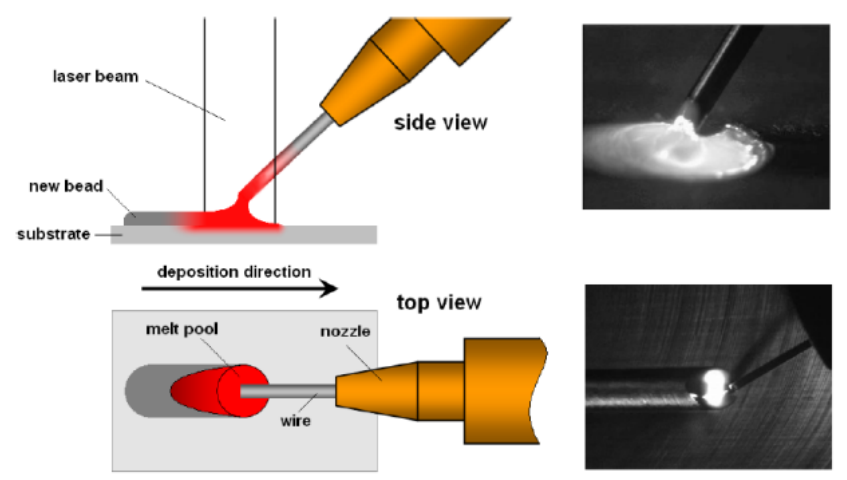

Figure 1. Left: Illustration of the laser-wire interaction. The molten metal solidifies into a bead by relative motion of the welding tool and the substrate. Right: Side- and top view images of the real process.

In Figure 2 the finished specimen, including the fixture can be seen. The building sequence is also indicated. The dimension of the substrate is $130 \times 100 \times 14 \mathrm{~mm}$ and the added material forms an open box with the dimension of $100 \times 70 \mathrm{~mm}$ and with a thickness of $4 \mathrm{~mm}$. 20 layers of material is added, each with a thickness of $0.7 \mathrm{~mm}$ giving a total wall height of $14 \mathrm{~mm}$. During the process an LVDT-gauge is used to measure the out-of-plane deformation at the center of the bottom surface of the substrate. Six thermocouples are attached to the substrate. Five of them are positioned close to the weld $(2,3,4,5$ and $6 \mathrm{~mm}$ from the outer weld toe). The sixth thermocouple is positioned at the bottom of the plate, close to the LVDT-gauge.

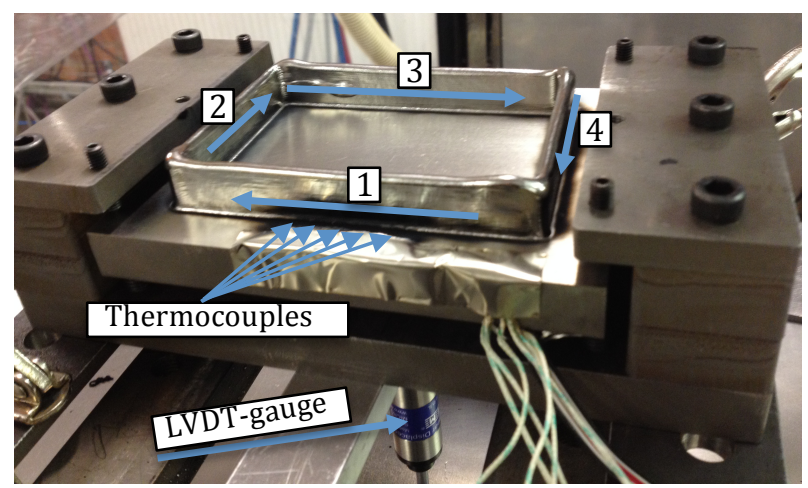

Figure 2. Finished specimen including the fixture and building sequence.

\section{Residual Stress Measurements}

The residual stress measurements were carried out by energydispersive X-ray diffraction on the high-energy beam line ID15A at the European Synchrotron Research Facility (ESRF) in Grenoble, France. The characterization used a high-flux white beam with an energy range of 50-250 keV. Measurements were done in transmission mode, with two energy-discriminating detectors positioned at $5^{\circ}(2 \theta)$ horizontally and vertically from the beam direction. The setup allows collection of complete energyintensity spectrums, which are converted to intensity vs. $2 \theta$ by the equivalent wavelength. A slit size of $100 \times 100 \mu \mathrm{m}^{2}$ was used for both the incident and diffracted beam slits, giving a diamond shaped gauge volume with a length of approximately $2 \mathrm{~mm}$.

The residual stresses were measured in the middle of one of the long walls of the box from to directions, by illumination from the side of the wall (giving strains in the $x$ and $z$ directions) and from the bottom side of the plate (giving strains in the $x$ and $y$ directions). During illumination from the side the gauge volume was placed in the second wall, and the incident beam was allowed to penetrate the first wall. The collection time for each measurement point was $60 \mathrm{~s}$, and the specimen was moved between measurements in order to perform line scans of the strains as a function of the position from the top of the wall. While collecting the spectrum during side illumination the specimen was moved back and forth $\pm 1 \mathrm{~mm}$ in the $x$ direction compared to the nominal position of the measurement to increase the gauge volume and statistics. Three parallel line scans were performed, spaced $2 \mathrm{~mm}$ apart to check repeatability. During illumination from the bottom, only one line scan was performed.

Spectrum fitting was performed using the General Structure Analysis System software [1] (GSAS) in order to determine the lattice parameters, $a$ and $c$, of the hcp $\alpha$-phase. Because of the low amount of $\beta$-phase in the structure, this was neglected in the present study. The average corresponding lattice strain was calculated according to [2]

$$
\epsilon_{\alpha}=\frac{2\left(a / a_{0}-1\right)+\left(c / c_{0}-1\right)}{3}
$$

where $a_{0}$ and $c_{0}$ are the unstrained lattice parameters. The unstrained parameters were in turn measured as a function of distance from the top of the wall on a $1 \mathrm{~mm}$ thick slice electric discharge machined (EDM) from a separate box manufactured with identical parameters. The residual stresses were calculated from the strains in the different directions as

$$
\sigma_{i}=\frac{E}{(1+\nu)(1-2 \nu)}\left[(1-\nu) \epsilon_{i}+\nu\left(\epsilon_{j}+\epsilon_{k}\right)\right]
$$

and permutations thereof (where $i, j, k=x, y$ or $z$ ).

In the analysis, the measurements of the unstrained lattice parameters showed a very large dependence of the position, most likely because the residual stresses were not completely relaxed in the slice. Estimated average values of $a_{0}=\mathrm{nm}$ and $c_{0}=\mathrm{nm}$ were used in the subsequent strain calculations, but the large uncertainty in the unstrained parameters is a major source of uncertainty in the residual stresses. Additionally, large columnar prior beta grains rendering in a strong texture from the AM process are expected in the wall, which further adds to the uncertainty.

\section{Computational Model}

The computational model applied in this work is a coupled thermo-mechanical finite element model. The coupling is done with the staggered approach and a large deformation formulation is applied. The software that is used for the simulations is the finite element software MSC.Marc. The FE-model can be seen in Figure 3. It contains 68280 elements and 80757 nodes. The elements that belong to the added material are initially deactivated in the model. They are then activated sequentially in each time 
step in the simulation. The size (volume) and position of the activated elements are determined with respect to the feeding wire diameter and feeding rate. A thorough description of how this is done can be found in [3].

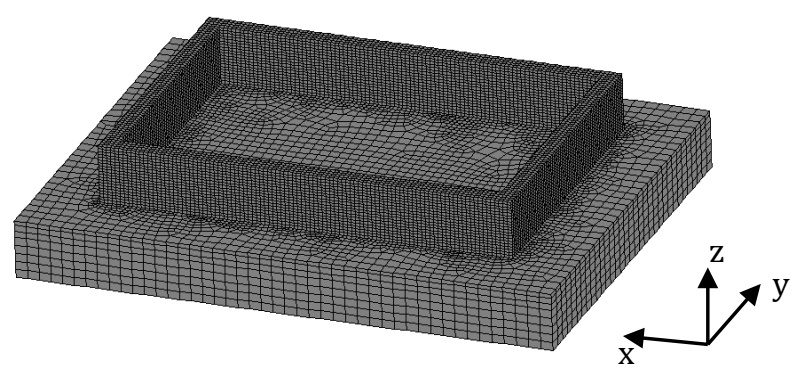

Figure 3. FE-model including substrate and added material.

The heat input is provided by a modified ellipsoidal heat source. The geometrical shape is elliptic in the plane but with a linear reduction in size in the depth. The distribution is similarly Gaussian in the plane but with a linear decrease through the depth [4]. This has proven to give a good representation of the heat distribution for high-energy beam sources, including the relative de-focused laser beam that is used in LMD. The heat source parameters that are used in this simulation are shown in Table 1. The heat loss to the surrounding was modeled as a convective part and a radiative part. The convection coefficient was set to 12 $\mathrm{W} / \mathrm{m}^{2} \mathrm{~K}$ and the emission coefficient to 0.05 . The mechanical boundary conditions are set to mimic the "simply supported" condition obtained by the four sharpened screw-pair shown in Figure 2. That is, the node in the upper left corner is restrained in all directions. The node in the lower left corner is restrained in the $\mathrm{x}$ - and $\mathrm{z}$-direction, while the nodes in the upper and lower right corners are restrained only in the z-direction.

Table 1. Heat source parameters. The net heat input, $Q_{n e t}$, has the unit $(\mathrm{W})$, the velocity, $v,(\mathrm{~mm} / \mathrm{s})$ and the rest of the parameters are in $(\mathrm{mm})$.

\begin{tabular}{|l|l|l|l|l|l|l|l|}
\hline Parameter & $Q_{\text {net }}$ & $v$ & $a$ & $b$ & $c_{f}$ & $c_{r}$ & $d$ \\
\hline Value & 912 & 10.0 & 2.5 & 0.6 & 2.0 & 2.0 & 0.75 \\
\hline
\end{tabular}

The material model that has been used is a physically based material model [5]. Physically based material models are formulated by consideration of the physics causing the deformation, dislocations and vacancies, and not only a curve fitting procedure for a measured stress- strain curve. It is assumed that physical based models do have a larger domain of applicability and can be extrapolated outside the calibration range provided they capture the dominant deformation mechanisms. This kind of model can have a natural coupling to evolution equations for phase changes, grain growth, precipitate growth etc. In this work an evolution model to predict the phase content is applied [6].

To reduce the computational time, parallel computation is utilized. The model is divided into 8 subdomains and each submodel is solved as a separate process. The computational time is approximately $96 \mathrm{~h}$, and this includes 6900 time increments. The actual process time for building the 20 layers is approximately 1.5 h.

\section{Results and Discussion}

Figure 4(a) shows the measured strains in the different directions. The strains in the $z$ direction agree well between the different line scans, whereas there are significant differences in the $x$ direction. In the following analysis, the average strains in the $x$ and $z$ directions from the three scans are used. The statistics and reliability of the measured strains in the $y$ direction is much lower since the long axis of the gauge volume coincides with the expected long axis of the large columnar grains. Therefore, only a few measurement points lead to convergence during peak fitting. In the evaluation, a $3^{\text {rd }}$ degree polynomial was fitted to converged points. The strains from the numerical simulations are also included in the figure, showing good agreement in the general trends. The magnitude of $\varepsilon_{x}$ is considerably higher in the simulations, whereas the lower (more negative) stresses are predicted in the $y$ direction compared to the experiments.

The resulting residual stresses are shown in Figure 4(b), which also includes the results from the numerical simulations. As expected from the strains in Figure 4(a), the overall trends are in agreement between experiments and predictions, whereas the levels differ. The largest residual stresses are observed in the $x$ direction, whereas the stresses in both the $y$ and $z$ directions are close to zero.
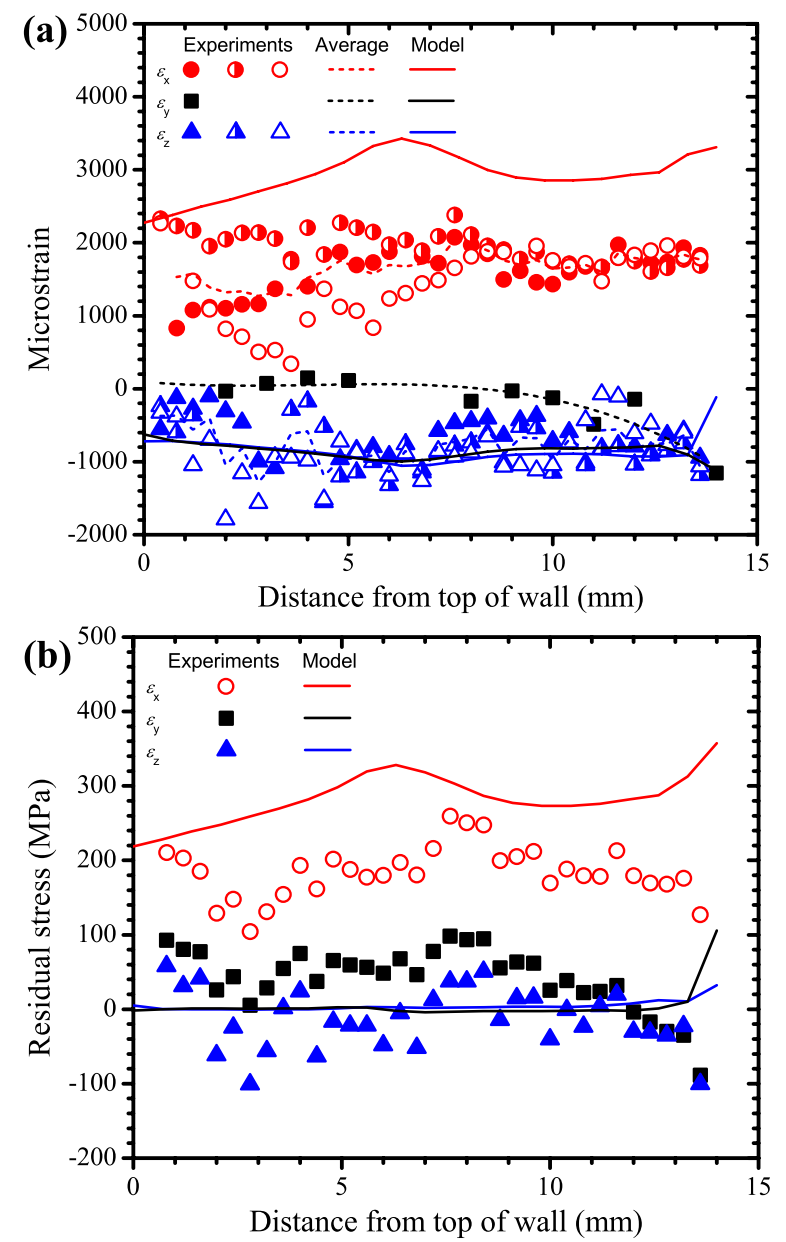

Figure 4. (a) Measured and predicted strains. (b) Measured and predicted residual stresses. 
In Figure 5 the measured and computed deformations is shown. The first figure shows the out of plane deformations during the entire process. One peak corresponds to the addition of one layer. It can be seen that the overall trend is that the results is diverging. The computed deformation is also over predicted in each layer. In Figure $5 \mathrm{~b}$ ), the first 800 seconds are shown. A close-up on the first $100 \mathrm{~s}$ is also included. One can here see that the model captures the shifts in deformation for all the steps in the process in quite detail (sequence 1, 2, 3, 4 and cooling until the next layer is laid). But nevertheless, in overall, there is an error that grows systematically.
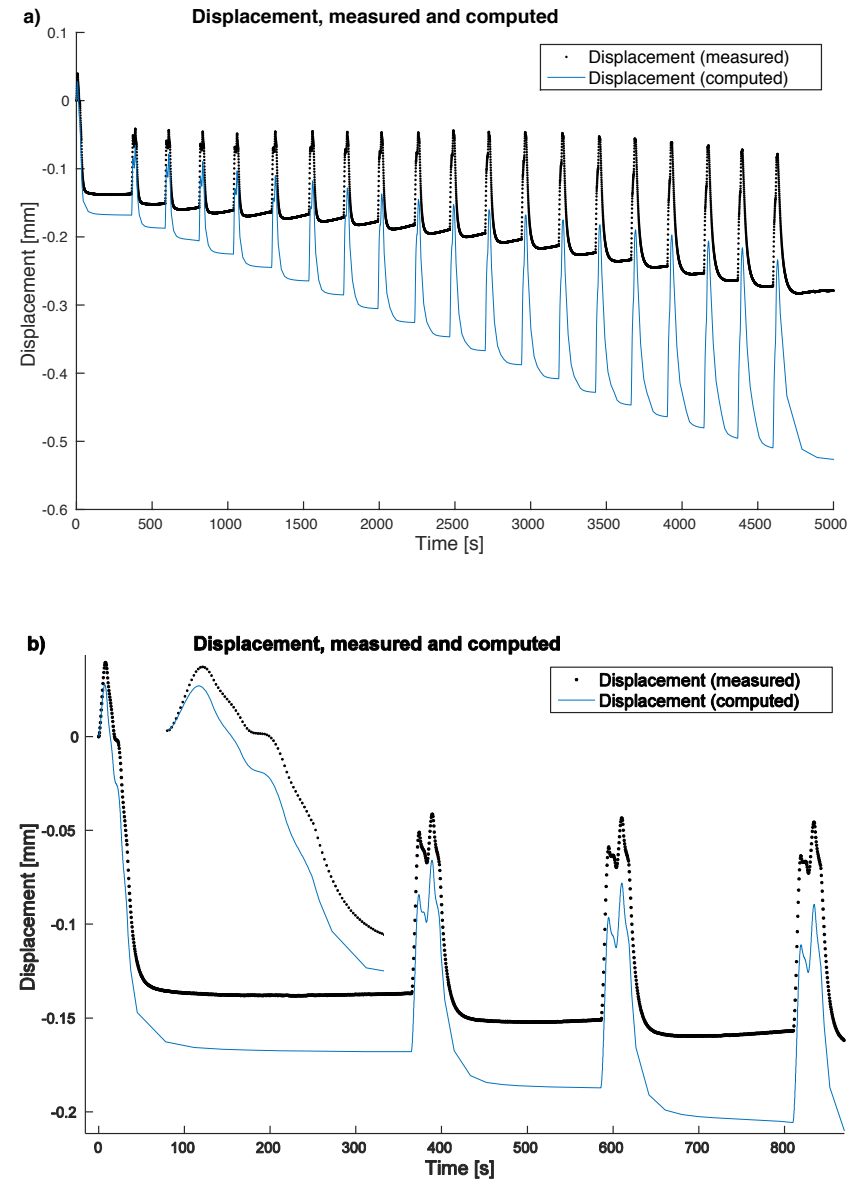

Figure 5. (a) Measured and predicted deformation history for the entire process, measured at the center of the bottom surface. (b) Close-up only showing the first $800 \mathrm{~s}$.

The measured and computed temperature history can be seen in . The agreement is over all very good, typically less than $5 \%$. The difference is a bit larger, about $10 \%$, at the very peaks when the heat source is just passing the measuring point.

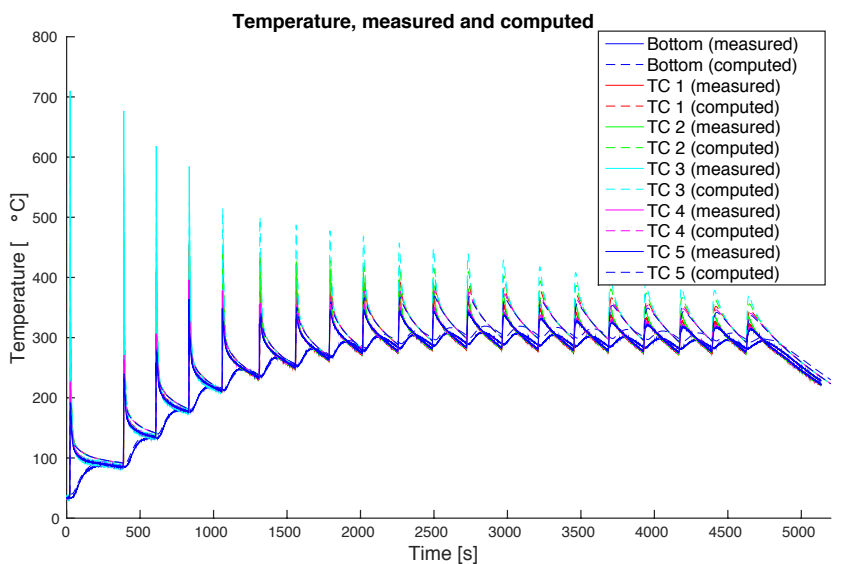

Figure 6. Measured and predicted temperature history.

\section{Acknowledgement}

The financial support was provided by the Swedish National Space Research Program (NRFP2). The beam line time at the European Synchrotron Radiation Facility (ESRF), ID15A in Grenoble, France, is greatly acknowledged. The work was performed in cooperation with GKN Aerospace Engine Systems, Trollhättan, Sweden.

\section{References}

1. A.C. Larson and R.B. von Dreele, General Structure Analysis System (GSAS). LANL Report No. LAUR 86748, Los Alamos National Laboratory, Los Alamos, NM, 2000, http://www.ncnr.nist.gov/xtal/software/ gsas.html

2. M.R. Daymond, M.A.M. Bourke, and R.B. von Dreele, "Use of Rietveld refinement to fit a hexagonal crystal structure in the presence of elastic and plastic anisotropy", J. Appl. Phys., 85 (1999), 739-747.

3. A. Lundbäck, and L.-E. Lindgren, "Modelling of metal deposition”, Finite Elem. Anal. Des., 47 (2011), 11691177.

4. A. Lundbäck, and H. Runnemalm, "Validation of threedimensional finite element model for electron beam welding of Inconel 718", Sci. Technol. Weld. Join., 10 (2005), 717-724.

5. B. Babu, and L.-E. Lindgren, "Dislocation density based model for plastic deformation and globularization of $\mathrm{Ti}$ 6Al-4V", International Journal of Plasticity, 50 (2013), 94-108.

6. C. Murgau, R. Pederson, and L.-E. Lindgren, "A model for Ti-6Al-4V microstructure evolution for arbitrary temperature changes", Modelling and Simulation in Materials Science and Engineering, 20 (2012). 